Original Article

\title{
Comparison of hamstring-to-quadriceps ratio between accelerating and decelerating sections during squat exercise
}

\author{
WON-GYU YOO ${ }^{1)}$ \\ 1) Department of Physical Therapy, College of Biomedical Science and Engineering, Inje University: \\ 607 Obangdong, Gimhae, Gyeongsangnam-do 621-749, Republic of Korea
}

\begin{abstract}
Purpose] The aim of this study was to compare hamstring-to-quadriceps ratio between the accelerating and decelerating sections for anterior cruciate ligament protection during squat exercise. [Subjects and Methods] Nine asymptomatic males were enrolled in this study. The hamstring (medial part) and quadriceps (rectus femoris) muscle activities during squat exercise were measured, and the squat exercises were classified into two sections (accelerating and decelerating) by using an accelerometer. [Results] The hamstring-to-quadriceps ratio was significantly higher in the decelerating section than in the accelerating section during the squat exercise. [Conclusion] Application of an increasing decelerating section strategy during the squat exercise can prevent damage in patients with a weakened anterior cruciate ligament due to sports activities.

Key words: Anterior cruciate ligament, $H / Q$ ratio, Squat exercise
\end{abstract}

(This article was submitted Mar. 16, 2016, and was accepted May 23, 2016)

\section{INTRODUCTION}

Decreased quadriceps muscle strength due to anterior cruciate ligament (ACL) tears is regarded as universal and is thought to be a compensatory mechanism to prevent anterior subluxation ${ }^{1)}$. Hamstring muscle strength, however, is relatively preserved because the hamstring can be facilitated or activated in response to an ACL tear ${ }^{2}$. Therefore, the balance between quadriceps and hamstring muscle strengths is usually assessed as hamstring-to-quadriceps $(H / Q)$ ratio $^{1-3)}$. Closed chain exercise does not cause anterior shear force because of the cooperative contraction of the quadriceps and hamstring in the patellofemoral joint, and functional muscle recruitment patterns can be provided as many joints move ${ }^{3)}$. This study compared $H / Q$ ratio between the accelerating and decelerating sections during the squat exercise for ACL protection.

\section{SUBJECTS AND METHODS}

Nine 9 asymptomatic males aged $25.4 \pm 3.2$ years (mean \pm SD) were enrolled. The subjects' mean height and weight were $176 \pm 2.5 \mathrm{~cm}$ and $69.5 \pm 4.7 \mathrm{~kg}$, respectively. All the subjects were healthy and had no back pain for a minimum of 1 year before the study. They had no lower limb or spine pathologies, and no rheumatological or neurological conditions. The purpose and methods of the study were explained to the subjects before obtaining informed consent for participation, according to the principles of the Declaration of Helsinki. Electromyography (EMG) signals were sent to the data acquisition unit of an MP150 system (BIOPAC Systems, Santa Barbara, CA, USA). EMG data were analyzed by using the AcqKnowledge version 3.9.1 software program and expressed as maximum voluntary contraction. Surface electrodes were attached on the hamstring (medial part) and quadriceps (rectus femoris), and the $H / Q$ ratio was calculated. The squat exercise involved descending to a $90^{\circ}$-knee flexion and ascending to the initial position at individual natural speed. A guide bar controlled the $90^{\circ}$-knee flexion 
of the habitual usual-speed squat exercise. The squat exercises were divided into two sections (accelerating and decelerating) by using an accelerometer. The data were analyzed for significant differences in $H / Q$ ratio during the squat exercise, using SPSS version 12.0 (Chicago, IL, USA). An independent $t$ test was performed to determine the significant differences in the activity ratios for each section during the exercises. A p value of $<0.05$ indicated statistical significance.

\section{RESULTS}

The $H / Q$ ratio was significantly higher in the decelerating section $(0.79)$ than in the accelerating section $(0.54 ; \mathrm{p}<0.05)$ during the squat exercise.

\section{DISCUSSION}

In conclusion, the quadriceps and hamstring showed strong tendency as antagonist and agonist muscles, respectively, in the accelerating section during knee flexion and reextension. These two muscles show stronger tendency as synergist muscles in co-contraction in the decelerating section. The squat exercise before that one was an isotonic exercise; however, the last squat was an isometric exercise ${ }^{4}$. Although isometric squat exercise aims to increase the $H / Q$ ratio, most players perform isotonic squat exercise to reinforce muscle strength in the lower limb. Isotonic squat exercise can induce future ACL injuries because excessive quadriceps muscle strength puts too much stress on the $\mathrm{ACL}^{3,4)}$, which decreases the $H / Q$ ratio. However, results of this study show that performing isotonic squat exercise that uses the decelerating section can enhance the $H / Q$ ratio. Application of an increasing decelerating section strategy during squat exercise can prevent damage in patients with a weakened ACL due to sports activities. Yoo reported that an effectively slow movement speed is recommended for selective strengthening of the vastus medialis oblique by using a slow speed-targeting device that provides biofeedback ${ }^{5)}$. Therefore, the squat exercise using the decelerating section can be easily applied if knee target or feedback on speed can be provided.

\section{REFERENCES}

1) de Jong SN, van Caspel DR, van Haeff MJ, et al.: Functional assessment and muscle strength before and after reconstruction of chronic anterior cruciate ligament lesions. Arthroscopy, 2007, 23: 21-28, 28.e1-28.e3. [Medline] [CrossRef]

2) Lee DH, Han SB, Lee JH, et al.: Quadriceps strength and endurance after posterior cruciate ligament tears versus matched group with anterior cruciate ligament tears. Arthroscopy, 2015, 31: 1097-1101. [Medline] [CrossRef]

3) Escamilla RF, Fleisig GS, Zheng N, et al.: Effects of technique variations on knee biomechanics during the squat and leg press. Med Sci Sports Exerc, 2001, 33: 1552-1566. [Medline] [CrossRef]

4) Sahrmann S: Diagnosis and treatment of movement impairment syndromes. New York: Mosby, 2002.

5) Yoo WG: Effects of the slow speed-targeting squat exercise on the vastus medialis oblique/vastus lateralis muscle ratio. J Phys Ther Sci, $2015,27: 2861-2862$. [Medline] [CrossRef] 